DESY M-83-02

February 1983

COMMENT ON WAKE FIELD COMPUTATION IN TIME DOMAIN

by

T. Weiland 
DESY behält sich alle Rechte für den Fall der Schutzrechtserteilung und für die wirtschaftliche Verwertung der in diesem Bericht enthaltenen Informationen vor.

DESY reserves all rights for commercial use of information included in this report, especially in case of filing application for or grant of patents.

"Die Verantwortung für den Inhalt dieses Internen Berichtes liegt ausschließlich beim Verfasser" 


\title{
COMMENT ON WAKE FIELD COMPUTATION IN TIME DOMAIN
}

\author{
by
}

T. Weiland

\begin{abstract}
A bunch of charged particles passing an accelerating cavity -or any other structure of varying shape- excites electromagnetic fields that act back on the particles. These wake fields are functions of space and time coordinates. The net change in momentum due to such fields is obtained by an integration over all times during that the fields interact with the particles, i.e. normally from $t=-\infty$ to $t=+\infty$. For ultrarelativistic particles passing structures of cylindrical symmetry this infinte range for the integration -which is very inconvinient for all practical computations-can be replaced by a finite range over the section where the scattered fields originate from.
\end{abstract}

\section{INTRODUCTION}

When a bunch of charged particles traverses an accelerating cavity electromagnetic fields are excited that lead to decelerating and deflecting forces acting back on the exciting particles. These fields are usually called wake fields and are functions of space and time. Since many accelerators and storage rings suffer significantly from such forces computational means have been developed for solving Maxwell's equations in time domain including the presence of free moving charges $/ 1 / 2 / 3 / 4 / 5 / 6 /$.

A typical situation is shown in figure 1: An accelerating cavity with side tubes of radius a. The actual shape of the cavity in the region $r>a$ is of no importance for the following considerations. Particles traversing the cavity from region A to $\mathrm{C}$ excite electromagnetic fields near region $\mathrm{B}$. These fields will in general remain only partly in region. B. For the PETRA cavity it was found/ 7 / that a significant amount of electromagnetic energy is radiated into the side tubes i.e. regions A and C. This fact implies that the interaction between the exciting particles and the fields is not limited to region B. Generally one has to take into account all the interactions taking place in the side tubes $A$ and $C$ (i.e. for $\mathrm{V}=\mathrm{c}$ particles only the exiting side tube region $\mathrm{C}$ ). The amount of interaction taking place in the side tubes increases the shorter the bunches are since 
bunches shorter than the tube radius contain many Fourier components above the cut off frequency of the side tubes. In linear accelerators and storage rings bunches can be in fact much shorter than the cavity hole radius. Even if they were not one would still be interested in the wake fields of very short bunches for instability studies since these short bunch wakes can serve as Green's function in tracking codes/8/. These facts represent a strong limitation to BCI type of computer codes since for short bunches one has to extend the mesh for the exiting side tube further and further the shorter the bunches are. Thus the mesh size increases roughly linearly with 1 /bunch length and the computation time quadratically. Due to the finite frequency resolution of a finite grid the ratio of r.m.s. bunch length over mesh step size must be at least of the order of five/1/2/3/4/. This condition leads to an additional increase of mesh size and computation time. Both effects seem to limit quite severly the applicability of BCI-like programs to very short bunches in open structures $/ 9 /$.

Since particle motion is considered to be describable by functions that vary slowly compared to the structure length of regions such as cavity gaps one uses in beam stability considerations (so far) only the integrated net change in momentum due to the self fields. The integrated wake fields are then called wake potentials. The formalism of impedances as used in accelerator theory is also connected (by simple Fourier transformations) with these integrated forces. These wake potentials can also be computed in frequency domain by means of eigenmode expansion/10/although only for the special case of an infinitely repeating chain of pill box cavities with connecting beam tubes/11/12/.

There exist two different solutions to the problem of short bunches in time domain computations of integrated forces that will be discussed in the following sections.

\section{WAVEGUIDE MODE EXPANSION}

Recently a solution has been described/6/13/ that solves for the exiting side tube fields by means of waveguide mode expansion. At some location in the downstream beam pipe the fields computed with BCI are matched to eigensolutions of the empty waveguide. These eigensolutions can then be integrated separately and the BCI calculation can stop at the matching location.

The disadvantage of this method is that additional computations and matching integrals over Bessel functions have to be invoked, for details see /6/13/. Furthermore this method is restricted to "normal" cavities with the beam hole at the center, i.e. cavities where the beam path area 
includes the symmetry axis $r=0$. Structures of coaxial type (e.g. wake field transformers/14/) where the beam offset $r_{1}$ is restricted by $0<R_{1} \leqq r_{1} \leqq R_{2}$ would invoke Bessel and Neumann functions including numerical procedures for searching the zeros of the nonlinear eigenvalue equations given by the boundary condition at two different radii. The waveguide mode expansion method has also not been applied to fields with higher azimuthal dependence, i.e. quadrupole sextupole and higher order fields, since DBCI/6/ solves only for dipole fields of bunches on axis having a dipole moment. Although monopole and dipole fields seem to suffice for beam stability considerations in storage rings it has been found that quadrupole wake fields represent a fundamental limitation to linear accelerators even if they were perfectly aligned/15/. A solution that is valid for any azimuthal mode number $m$ and general structures is described next.

\section{INTEGRATION AT THE BEAM TUBE SURFACE}

Another solution to the exiting side tube problem/16/ has been implemented into TBCI/5/. This method is not restricted to monopole and dipole fields and also not to cavities with an on-axis beam hole. The method does not invoke any additional effort in the computation but makes use only of the knowledge about the explicitly and analytically known dependencies of the integrated forces as a function of radius and azimuth.

Consider a particle at radius $r_{1}$ passing a structure from left to right at the speed of light and at $r_{2}$ a second particle at the distance s as shown in figure 1. The net change in momentum experienced by the second particle due to the fields generated by the leading particle can be generally written as:

$$
\begin{aligned}
& \Delta \vec{p}\left(r_{1}, r_{2}, \varphi_{1}, \varphi_{2}, s\right)=e \sum_{m=0}^{\infty} \text {. }
\end{aligned}
$$

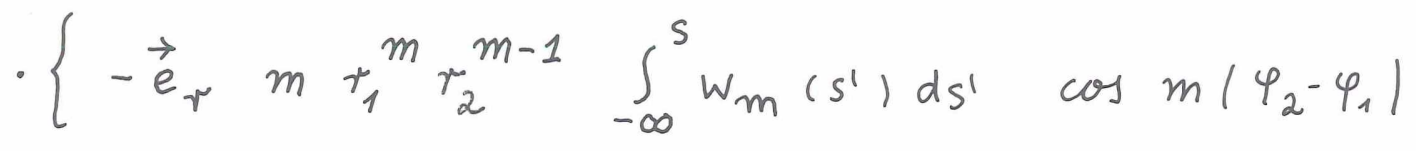

$$
\begin{aligned}
& +\vec{e}_{\varphi} m r_{1}^{m} r_{2}^{m-1} \int_{-\infty}^{s} w_{m}\left(s^{\prime}\right) d s^{\prime} \sin m\left(\varphi_{2}-\varphi_{1}\right) \\
& \left.+\vec{e}_{z} \tau_{1}^{m} \tau_{2}^{m} \quad w_{m}(s) \quad \cos m\left(\varphi_{2}-\varphi_{1}\right)\right\}
\end{aligned}
$$

This relation is known for infinitely repeating structures/17/18/ and for single cavities in the case of fields with no azimuthal dependence/19/ (i.e. 
$m=0$ ). It is shown briefly in the Appendix that this relation holds for single cavities and any azimuthal dependence as well. It obviously does not matter where the forces are integrated since the forces at any radial and azimuthal position can be expressed by the forces at any other position $r, \varphi$. The wake functions $\mathrm{w}_{\mathrm{m}}(\mathrm{s})$ are characteristic functions of the structure and have to be calculated by solving Maxwell's equations for the given geometry and initial conditions. They are essentially given by the net change in longitudinal momentum as:

$$
W_{m}(s)=\int_{-\infty}^{\infty} E_{z m}\left(r_{2}, \varphi_{2}, z=c t-s\right) d t /\left(r_{1}^{m} r_{2}^{m} \cos m\left(\varphi_{2}-\varphi_{1}\right)\right)
$$

It is interesting to notice that for each $m$ the transverse force and the longitudinal force are simply related and given by a single function $\mathrm{w}_{\mathrm{m}}(\mathrm{s})$. This reflects the well known relation between transverse and longitudinal force in frequency domain/20/:

$$
\vec{\sim}_{\perp \perp}=\frac{-i}{\omega} \vec{\nabla}_{\perp} E_{z}
$$

For a cavity as shown in figure 1 the original integral $I_{1}$ for the longitudinal force seen by a particle near the axis can be replaced by the integral $\mathrm{I}_{2}$ for a test particle traversing the cavity at the surface of the beam tubes:

$I_{1}=$

$$
\int_{-\infty}^{+\infty} E_{z_{m}}\left(r_{2} \ll a, \varphi_{2}, z=c t-s, t\right) d t \quad r_{2}^{m}
$$

(4)

$$
I_{2}=\int_{-\infty}^{+\infty}=\int_{-g / 2}^{+g / 2} E_{z m}\left(r_{2}=a, \varphi_{2}, t=c t-s, t\right) d t / a^{m}
$$

The second integral extends only over the cavity opening (region B) since the longitudinal force vanishes at the tube surface. As a consequence the field computation can stop as soon as the last test particle reaches the exiting side tube. Furthermore the fields have to be computed only in the region $B$ where they are generated. As a consequence there is no need to extend the mesh and the time range of computation to a long exiting side tube. 
There are two more useful consequences from the above considerations that help to ease the wake potential computation in time domain.

It can be seen that for $r_{1}=r_{2}=a$ fields are excited first when the particles leave the left end of the opening. From this moment on scattered fields can travel towards the outermost ends of the mesh. If the way forth and back from the opening to the ends of the mesh is longer than the total range in $s$ for which $w_{m}(s)$ is computed, fields scattered at the end of the mesh cannot reach the test particles before they enter the right end of the opening. This implies that under these conditions the boundary conditions at the mesh ends in the field computation do not influence the wake potential $\mathrm{w}_{\mathrm{m}}(\mathrm{s})$. This follows directly from causality principle.

For bunches near the axis it was observed/1/3/4/ that a numerical noise is coming into the wake calculation even in a smooth pipe -which does not produce any wake force in theory-. This tube contribution has to be computed separately and subtracted from the original wake/8/. When test particles travel along a pipe at the surface there can be no force in the computations either since the forces parallel to the pipe surface are set zero in the mesh. Thus the problem of numerical pipe wake fields is solved too by integrating the force at $r_{2}=a$.

For coaxial structures similiar expressions can be derived for the wake functions but details are left to the Appendix and a later paper.

\section{CONCLUSION}

The explicit knowledge of the forces experienced by particles at different radial positions passing a cavity at the speed of light enables to transform the infinte time range of the electromagnetic interaction to a finite interval. For field computations in the time domain this implies that there is no need to evaluate the downstream fields travelling with the particles away from a cavity. Although these fields interact with particles near the axis somewhere in the downstream beam pipe the net change in momentum can be computed from fields experienced by test particles at the beam tube surface. The interaction for these test particles is limited to the cavity opening since no forces exists parallel to the surface of the beam tubes.

\section{ACKNOWLEDGEMENTS}

The author thanks F.Willeke for many helpful and clarifying discussions. 


\section{APPENDIX}

Considering a cavity as shown in figure 1 or 2 one can write for the electric field in the region $R_{1} \leqq r \leqq R_{2}$-independant of the actual shape of the disturbence for $r>R_{2^{-}}$

(A1) $\overrightarrow{\mathrm{E}}(r, \varphi, z, t)=\operatorname{Re}\left\{\frac{1}{2 \pi} \int_{-\infty}^{+\infty} d \omega e^{i \omega t} \sum_{m=-\infty}^{+\infty} e^{i m \varphi} e^{-i k(\omega) z} \vec{g}_{m}(\omega, r)\right\}$

with:

(A2) $\quad \mathrm{k}(\omega) \quad$ propagation constant in $\mathrm{z}$ direction

m azimuthal mode number

The net change in longitudinal momentum of a particle at $r=r_{2}$ at the speed $\mathrm{v}=\beta \mathrm{c}$ and at $\mathrm{z}=\beta \mathrm{ct}-\mathrm{s}$ is obtained from:

$$
\begin{aligned}
& \Delta P_{z}(r, \varphi, s)=\frac{e}{2 \pi} \operatorname{Re}\{ \\
& \left.\int_{-\infty}^{+\infty} d t \int_{-\infty}^{+\infty} d \omega e^{i \omega t} \sum_{m=-\infty}^{+\infty} e^{i m \varphi} e^{-i k(\omega) \beta c t} e^{i k(\omega) s} g_{m z}(\omega, r)\right\}
\end{aligned}
$$

The fields in the region above $R_{2}$ have to be matched to the fields in the region below such that the total field fulfills the boundary conditions and such that the two fields have continuous tangential components at the interface at $r=R_{2}$ inside the gap, i.e. the fourier coefficients $\vec{g}_{m}(\omega, r)$ have to be chosen properly. Boundary conditions also define the dispersion relation for the propagation constant $k(\omega)$. With:

$$
\delta(k(\omega) \beta c-\omega)=\frac{1}{2 \pi} \int_{-\infty}^{+\infty} e^{i(\omega-k(\omega) \beta c) t} d t
$$

and

$$
G_{m}(s, r)=\int_{-\infty}^{+\infty} e^{i k(\omega) s} g_{m z}(\omega, r) \delta(k(\omega) \beta c-\omega) d \omega
$$


the expression for the longitudinal change in momentum becomes

$$
\Delta P_{z}(\tau, \varphi, s)=e \operatorname{Re}\left\{\sum_{m=-\infty}^{+\infty} e^{i m \varphi} G_{m}(s, r)\right\}
$$

We now recall the relation between the transverse and longitudinal component of the force $\overrightarrow{\mathrm{F}}$ in frequency domain/20/:

$$
\begin{array}{ll}
\underset{\sim r}{F_{r}}=-\frac{\beta c}{i \omega} \frac{\partial}{\partial r} & e \underset{\sim}{E} \\
\underset{\sim}{F_{\varphi}}=-i \frac{\beta c}{i \omega} & \frac{m}{r} \quad \underset{\sim}{E_{z}} \\
\underset{\sim z}{F_{z}}= & e \underset{\sim}{E}
\end{array}
$$

which follows directly from Maxwell's equation in complex notation

$$
\text { i } \omega \vec{\sim} \vec{\sim}=\text { cure } \underset{\sim}{\vec{E}}
$$

for those frequencies that contribute to the interaction, i.e. if one uses:

$$
k(\omega) \beta c=\omega
$$

i.e. only those Fourier components are considered that contribute to the force.

Since it is possible to obtain the transverse forces from the longitudinal one we investigate the wave equation for the longitudinal component of the electric field:

(A10) $\frac{1}{c^{2}} \frac{\partial^{2} E_{z}}{\partial t^{2}}=\frac{\partial^{2} E_{z}}{\partial z^{2}}+\frac{1}{r^{2}} \frac{\partial^{2} E_{z}}{\partial \varphi^{2}}+\frac{1}{r} \frac{\partial}{\partial r}+\frac{\partial}{\partial r} E_{z}$

Using the above relation between the propagation constant and the frequency (A9) yields:

$$
\frac{1}{\gamma^{2} \beta^{2}}\left(\frac{\omega^{2}}{c^{2}} E_{z}\right)=\frac{-m^{2}}{r^{2}} E_{z}+\frac{1}{r} \frac{\partial}{\partial r} r \frac{\partial}{\partial r} E_{z}
$$

For ultrarelativistic particles we find from this $(\gamma->\infty)$ that $G_{m}(s, r)$ must be a solution of: 


$$
0=\frac{-m^{2}}{r^{2}} G_{m}(s, r)+\frac{1}{r} \frac{\partial}{\partial r} r \frac{\partial}{\partial r} G_{m}(s, x)
$$

i.e.:

$$
\begin{aligned}
& G_{0}(s, r)=u_{0}(s)+v_{0}(s) \ln r ; m=0 \\
& G_{m}(s, r)=u_{m}(s) r^{m}+v_{m}(s) r^{-m} ; m \neq 0
\end{aligned}
$$

Although this conclusion is strictly valid only for finite $\omega$ it still can be made bearing in mind that the result will be applied only to smooth partcile densities with a decaying frequency spectrum at very high frequencies.

In the general case of coaxial structures we have to use throughout the calculation both solutions of (A13) and all $\mathrm{m}$ 's in (A14). We will restrict here -for simplicity- our considerations to "normal" cavities with $R_{1}=0$. This implies that the logarithmic term in (A13) must vanish as well as all terms with a negative $m$ in (A14). Now that we now explicitly the radial functions we see from (Ay) that in time domain the net change in momentum can be written as:

$$
\begin{aligned}
& \overrightarrow{\Delta P}(r, \varphi, s)=e \sum_{m=0}^{\infty} \\
& \left\{-\vec{e}_{r} m r^{m-1} \int_{-\infty}^{s} u_{m}\left(s^{\prime}\right) d s^{\prime} \cos m \varphi\right. \\
& +\vec{e}_{\varphi} m r^{m-1} \int_{-\infty}^{s} u_{m}\left(s^{\prime}\right) d s^{\prime} \sin m \varphi \\
& \left.+\vec{e}_{z} r^{m} u_{m}(s) \quad \cos m \varphi\right\}
\end{aligned}
$$

The integral comes from the division by $i \omega$ in the relation between transverse and longitudinal forces in (A7).

The above relation describes the momentum change in energy due to fields that are present before the particle enters. From conservation of energy it follows that that the fields generated by the particles get the energy out of the longitudional momentum -since $r_{1}$ and $r_{2}$ were defined to be constant-. Thus it follows for the general case of a particle \#1 at $r=r_{1}$ generating the fields that act on a second particle \#2 at $r=r_{2}$ that the net change in momentum is given by equn (1). 

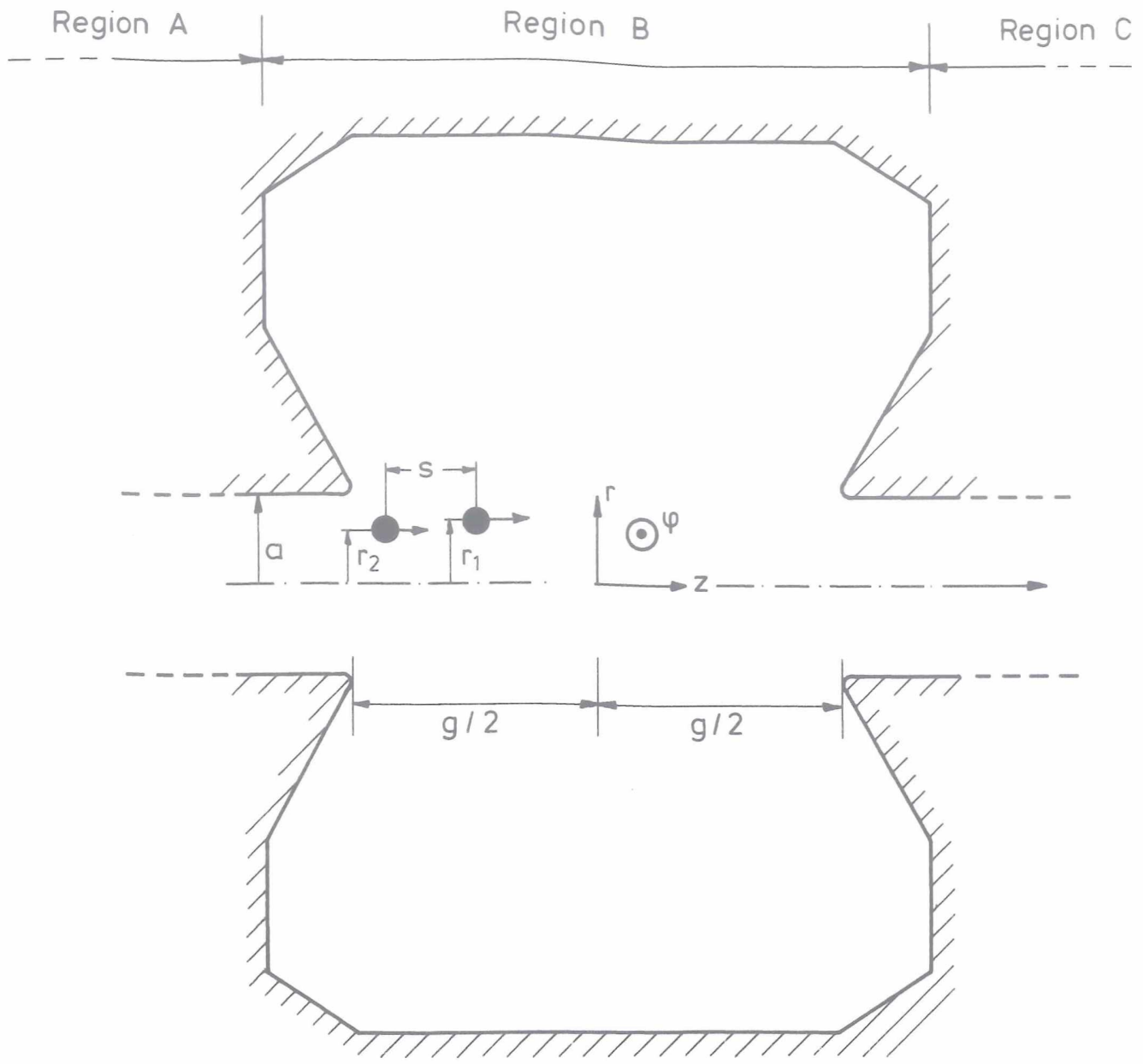

Figure 1 Cylindrically symmetric cavity with side tubes of radius a

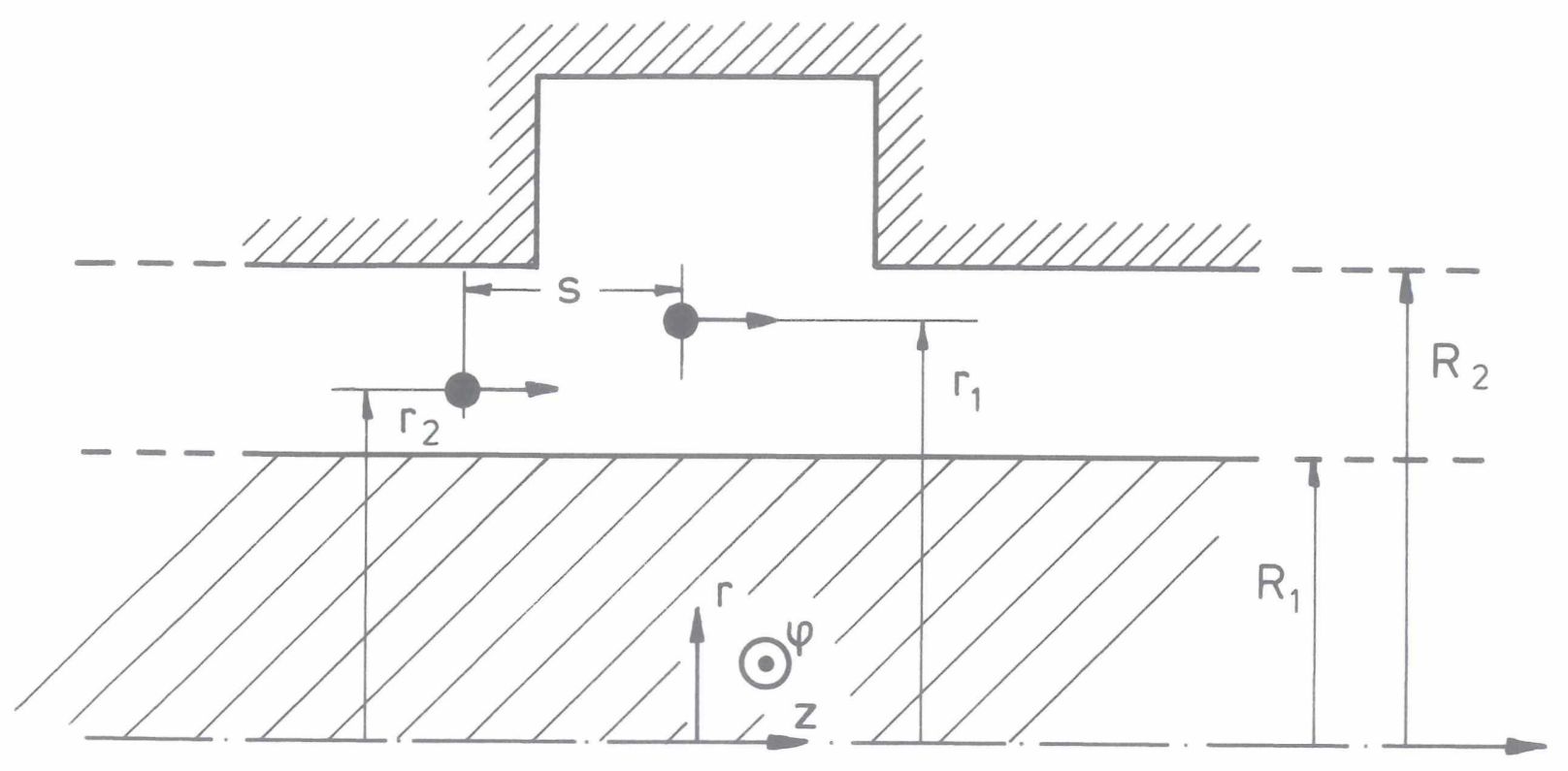

Figure 2: Coaxial cavity structure 


\section{LITERATURE}

/1/ T.Weiland, Proceedings of the XI-th International Conference on High Energy Accelerators, Geneva 1980,Birkhauser Verlag, Basel, edited by W.S. Newman, pp. 575

/2/ T.Weiland, CERN/ISR-TH/80-07, Januar 1980

/3/ T.Weiland, CERN/ISR-TH/80-45, November 1980

/4/ T. Weiland, CERN/ISR-TH/80-46, November 1980

/5/ T.Weiland, DESY 82 -015, March 1982, being reprinted in Nuclear Instruments and Methods in Physics Research(1983)

/6/ G.Aharonian et al., accepted for publication in Nuclear Instruments and Methods in Physics Research

/7/ T.Weiland, DESY M-81-04, April 1981

/8/ T.Weiland, DESY 81 -088, December 1981

/9/ T.Weiland, talk given at the International Workshop on Single Beam Instabilities held at Cornell in March 1981 and subsequent discussion

/10/ T.Weiland and B.Zotter, Particle Accelerators 11 (1981), pp. 143

/11/ E.Keil, Nuclear Instruments and Methods in Physics Research 100 (1972), pp.419

/12/K.Bane and B.Zotter, Proceedings of the XI-th International Conference on High Energy Accelerators, Geneva 1980,Birkhauser Verlag, Basel, edited by W.S.Newman, pp.581

/13/ A.Aharonian et.al, CBN 81-32, November 1981

/14/G.A.Voss and T.Weiland, DESY 82 -074, December 1982 and DESY M 82-10, April 1982

/15/ A. Chao and R.K. Cooper, SLAC-Pub 2945, June 1982

/16/ T.Weiland, letter to all TBCI/BCI users, August 1982

/17/ K.Bane and P.B.Wilson, Proceedings of the XI-th International Conference on High Energy Accelerators, Geneva 1980,Birkhauser Verlag, Basel, edited by W.S.Newman, pp.592

/18/ A. Chao, SLAC-Pub 296, August 1982

/19/ A.Piwinski, DESY-M-VM-80-16, May 1980

/20/ A.Panofsky and W.A.Wenzel, Rev.of Sci.Inst. 2y (1956), pp.967 